\title{
A model of spatially constrained social network
}

\section{dynamics}

\author{
Elisabeth zu Erbach-Schoenberg ${ }^{1,2}$, Seth Bullock ${ }^{1,3}$ and Sally Brailsford ${ }^{2}$, \\ ${ }^{1}$ Institute for Complex Systems Simulation, ${ }^{2}$ School of Management, and \\ ${ }^{3}$ School of Electronics and Computer Science, \\ University of Southampton, Southampton, UK \\ e.zuerbach-schoenberg@soton.ac.uk
}

October 10, 2013

\begin{abstract}
Social networks characterise the set of relationships amongst a population of social agents. As such, their structure both constrains and is constrained by social processes such as partnership formation and the spread of information, opinions and behaviour. Models of these coevolutionary network dynamics exist, but they are generally limited to specific interaction types such as games on networks or opinion dynamics.

Here we present a dynamic model of social network formation and maintenance that exhibits the characteristic features of real-world social networks such as community structure, high clustering, positive degree assortativity and short characteristic path length. While these macrostructural network properties are stable, the network micro-structure undergoes continuous change at the level of relationships between individuals. Notably, the edges are weighted, allowing for gradual change in
\end{abstract}


relationship strength in contrast to more abrupt mechanisms, such as rewiring, used in other models.

We show how the structural features that characterise social networks can arise as the result of constraints placed on the interactions between individuals. Here we explore the relationship between structural properties and four idealised constraints placed on social interactions: space, affinity, time, and history. We show that spatial embedding and the subsequent constraints on possible interactions are crucial in this model for the emergence of the structures characterising social networks.

\section{Introduction}

Patterns of human interactions are often characterised as social networks. In such networks, individuals are represented by network nodes and are connected by a network edge (a link) if they interact with each other in some way (Newman, 2010). The nature of the interactions being represented depends on the setting considered. Network connections might represent mere proximity between individuals, a history of explicit interactions of some type, e.g., sexual contacts (Liljeros et al., 2001), face-to-face meetings (Cattuto et al., 2010), phone calls (Onnela et al., 2007) or online interactions (Grabowski, 2007; Leskovec and Horvitz, 2008; Szell and Thurner, 2010). Or they might be limited to representing interactions with a specific function, such as one individual seeking advice from another. It is often appropriate to attribute a strength or weight to each network connection, since some social interactions may be stronger or take place more frequently than others (Barrat et al., 2004, 2005).

Because real social behaviour changes over time, social networks are inherently dynamic in nature. Each node's number of network neighbours (its degree), the identity of these neighbours as well as the strengths of the connections 
may change over time as a result of the underlying social processes, behavioural decisions and externalities that shape social interactions. It has been shown that incorporating the dynamics of the interaction structure in models of social behaviour influences the observed outcomes (Bravo et al., 2012).

Although the structure of a social network may tend to be constantly changing in terms of which individuals can be said to be connected to which others, the overall aggregate statistical properties of the network may be remarkably stable (Bryden et al., 2011). While individual nodes and edges appear and disappear over time, properties such as connectivity, clustering (how likely it is for individuals to have mutual friends), degree distribution or degree assortativity (correlation between the number of friends that an individual has and the number of friends its friends have) tend to remain characteristic of social networks in general (Newman, 2002; Toivonen et al., 2006; Wong et al., 2006).

While existing network models have tended to concentrate on generating a static topology that matches the statistical properties of some class of realworld network (Barrat et al., 2005; Toivonen et al., 2006; Wong et al., 2006), it would also be desirable for a dynamic model to exhibit these properties as a consequence of idealised ongoing social dynamics within the model.

Such a model would allow the exploration of research questions such as: To what extent do the stable aggregate properties of social networks arise from the local interactions between individuals? What properties must individuals have and be sensitive to in order for appropriate structures to form? How stable are these structures to perturbations of various kinds? In this paper we take some steps towards such a dynamic model of social network formation and maintenance.

There are of course a number of approaches that can be taken to model construction in this situation (Levins, 1966). Our aim for this paper is not to 
present an empirically calibrated model of a specific social process, but rather to present a simple heuristic or conceptual model (Oreskes et al., 1994) that reflexively links network-mediated processes of social interaction with socially mediated processes of network change. This style of model is not intended to allow us to make predictions about real social networks. Instead its aim is to allow us to better understand how different theoretically motivated constraints on social interaction might combine to influence the structure and dynamics of social networks.

Given that there are still relatively few models in this area, and those that do exist tend to concentrate on either strategic interactions between agents or spreading processes on social networks, it is appropriate to ask which (if any) typical properties of social networks tend to arise robustly from simple, local, network-mediated constraints on basic social contact, and which do not. To make sense of such a model we must build a bridging account that relates how local interactions give rise to network structure, and how network structure feeds back on local interactions. Such a bridging account can then be tested through directed empirical enquiry into real social networks.

In this paper we present a model that takes the first steps on this path, demonstrate the conditions under which it has appropriate system-level properties that arise from ongoing dynamic interactions and explain this relationship in terms of the parameterised constraints that govern system behaviour.

In the next section we locate our model with respect to existing network models that have relevance for social systems. Subsequently we specify our dynamic network model and report some results that describe its behaviour. We explain these results, and conclude with a discussion of the model and directions for future work. 


\section{Social Network Models}

As outlined in the previous section, we argue that a dynamic social network model should generate a social network where the low-level topological structure is subject to change as the result of ongoing processes that are idealisations of social behaviours, while the high-level statistical characteristics of the network topology, e.g., clustering or assortativity, are relatively stable and tend to lie within the range of values observed for the appropriate real-world social networks. This dynamic model approach contrasts with static network models which aim to efficiently grow or generate a fixed topology whose statistical properties are consistent with empirical social network data.

Real-world social networks are relatively sparsely connected and exhibit high levels of clustering, positive assortativity with respect to degree, community structure and short characteristic path length (meaning most individuals can reach most other individuals through very few intermediate contacts) (Newman, 2002; Newman and Park, 2003; Toivonen et al., 2006). There has been some debate as to whether the degree distribution in social networks follows a powerlaw or not, exhibiting a few nodes with very high degree even though most nodes have a low degree. This seems to depend on the interaction considered and data on communication and contact networks generally lack a power law's long-tail and if it is present there is a cut-off for higher values (Amaral et al., 2000). This is due to the fact that in these types of social networks, contacts require active maintenance in order to persist, in contrast to, e.g., online social networks, where after "friending" someone, the contact may persist without maintenance.

Many models that are able to grow networks that mimic social network structure exist and the networks resulting from these models match the characteristic topological features observed in real-world social networks. While some 
models of social networks grow the network in a dynamic fashion, the growth process is typically a means to achieve some class of final network structure rather than an attempt to model the human social behaviours that generate real social networks. One approach used in these models is to grow the network by connecting one node at a time to the already existing nodes until the desired network size has been reached (Holme and Kim, 2002; Toivonen et al., 2006; Vázquez, 2003). In real social networks new connections are often made when an individual brings two of their friends into contact, which as a result form a connection between them. This triangle closure is an important process and is used by all of the models discussed here to achieve strong clustering (meaning connected nodes are likely to have network neighbours in common (Newman, 2010). In other models the dynamics are a very high level abstraction of human behaviour but rely on periodic removal of randomly chosen edges or nodes (including all their edges) in order to maintain the system in a steady state (Davidsen et al., 2002; Ebel et al., 2002; Kumpula et al., 2007; Marsili et al., 2004). Models of coevolutionary or adaptive networks focus on the mutual feedback between processes constrained by the network and topological change as a result of individual behaviour (Gross and Blasius, 2008; Skyrms and Pemantle, 2000). These models rely on a specific process taking place on the network such as opinion dynamics or pairwise games (Eguíluz et al., 2005; Holme and Newman, 2006; Takács et al., 2008; Van Segbroeck et al., 2009) which restricts their applicability to the study of very specific social dynamics.

With the exception of Kumpula et al. (2007) and Skyrms and Pemantle (2000), all models described here do not allow for weighted edges, despite the fact that this is important if we want to model gradual changes of the topology, or represent so called weak ties (Granovetter, 1973).

An important factor in some models of social networks is spatial embedding 
of the nodes, often accompanied by spatial constraints that limit interactions to occur only between pairs of spatially proximal nodes (Boguñá et al., 2004; Wong et al., 2006). A model using a random geometric graph (Penrose, 2003) is presented in Hamill and Gilbert (Hamill and Gilbert, 2009). In their model a social reach of interaction is defined for each node as a circle of a certain radius centred on the node's spatial location and two nodes may only interact if their spatial reaches overlap. These models generate networks using spatial constraints but do not attempt to model dynamics.

In the model presented in this paper we adopt a similar spatial constraint on interaction, but also include idealised social interactions that allow for triangle closure. Furthermore, we explicitly model the dynamic change of the network as a result of mutual, coevolutionary feedback between the network topology and individuals' decisions and actions. The model's behavioural rules are designed such that they reflect certain aspects of human social behaviour. This allows us to understand how individual behavioural tendencies lead to macro-level topology and which factors of this behaviour are important for creating structure observed in the real-world.

\section{The $R A S H$ Model}

In this section we will introduce an algorithm to establish and maintain a dynamic social network using only local rules. We will introduce the algorithm and its relevant parameters and provide context for the modelling choices taken.

The core of the model is very simple. Each timestep, every individual invites their network neighbours to gatherings where connections between individuals are made and strengthened. This mode of social interaction allows for an individual's mutual friends to be introduced to each other, resulting in triangle closure. It also makes possible the maintenance of connections between people 
who would not actively maintain contact, which could be a result of constraints of distance or disinterest.

Four primary factors influence the design of the model, reflecting constraints that operate on real-world social interactions. Each factor is governed by a single model parameter, $R, A, S$ and $H$ :

1. Interactions are constrained by a limited interaction range: individuals separated by a spatial distance greater than $R$ cannot accept each others' invitations.

2. Interactions are constrained by affinity: with probability $A$ there is enough affinity between a pair of individuals to interact. With probability $1-A$ there is a lack of affinity and the individuals will not accept each others' invitations. They can however, meet if both follow a mutual friend's invitation.

3. Interactions are constrained by a limited number of time slots available: each individual can accept at most $S$ invitations per timestep.

4. Interactions are constrained by history: invitations tend to be accepted when the individuals involved have a strong history of interaction over the preceding $H$ timesteps.

We will describe these four constraints on the model in more detail before formally specifying the algorithm that implements them.

First, real-world social networks are embedded in space and many of the properties that define social networks are linked to spatial embedding (Barnett et al., 2007; Bullock et al., 2010; Herrmann et al., 2003). It has been shown that people are more likely to interact with individuals spatially close to them (Illenberger et al., 2012). We therefore embed nodes in space by assigning them a random position in a two-dimensional bounded square arena. Each individual 
may only invite another to its gathering if the distance between them is not more than $R$. This is similar to the spatial restriction used by Hamill and Gilbert (Hamill and Gilbert, 2009, 2010) and following them we will refer to it as the social reach or reach of an individual. In their model, two individuals interact if their reaches overlap therefore leading to an interaction distance of up to $2 R$. In addition to this constraint on direct interactions, individuals in our model can interact with friends of their friends, even if these individuals are located beyond their own direct social reach, since they can meet at a mutual friend's gathering. This relaxes the limitations on triangle closure present in Hamill's social reach model.

In addition to spatial distance, pairs of individuals may be separated by issues of personality or belief. To reflect this, each pair of individuals is assigned an affinity value describing how well they get along. This value is mutual and therefore symmetrical, and, once assigned, it remains constant for the duration of a run. For simplicity we only distinguish between two cases. With probability $A$, a pair of nodes have sufficient affinity to send invites to each other. With probability $1-A$, the pair's affinity is not sufficient for invitations to be sent.

For most social systems, social interactions are limited by the time budgets of the individuals involved (Miritello et al., 2013; Sutcliffe et al., 2012). In general, individuals have more opportunities for social interactions than time available to pursue these opportunities. We model this constraint by restricting individuals to attend at most $S$ gatherings per time step, chosen from amongst the (typically greater than $S$ ) invites that they receive. They choose based on affinity as well as relative familiarity (based on the previous encounters reflected in the edge weight), similar to the mechanism of choosing interaction partners described in the "Friends" models in Skyrms and Pemantle (2000).

Finally, real social connections must typically be actively maintained or they 
erode and eventually disappear (Cummings et al., 2006). A simple mechanism to achieve this is to use a global decay process which reduces all edge weights every time step. This can be difficult to calibrate, often leading to networks either fragmenting completely if decay is too strong, or connecting fully if decay is too weak.

Some models avoid this problem through occasionally removing nodes with all their edges. This can be an effective global mechanism. However, it does not allow for the gradual decay of connection strengths. Furthermore, this requires a turnover of nodes. Depending on the time-scales considered, this can be a realistic or unrealistic assumption. Here we focus on small groups of individuals over relatively short periods of time where it is more realistic to assume a fixed number of nodes.

Consequently, rather than implement decay or turnover processes, we have chosen a localised mechanism inspired by the fact that friendships need to be actively maintained. In this model each individual has a memory recording their meetings with other individuals for each of the previous $H$ time steps. The number of encounters between two individuals $i$ and $j$ at timestep $t$ is denoted $h_{i j}^{t}$ and can range from 0 to $S+1$ occasions per timestep, the maximum value corresponding to the situation where both individuals attend each others' gatherings and also attend the same $S-1$ gatherings hosted by their other shared neighbours.

If two individuals have had no meetings in the last $H$ timesteps, then there is no edge between them, otherwise an edge exists with a particular strength or weight. The weight of an edge between individuals $i$ and $j$, is calculated as $w_{i j}=\frac{1}{H} \sum_{t=1}^{H} h_{i j}^{t}$, summing the total number of meetings in the pairs recorded interaction history $h_{i j}$ and dividing this number by the history length $H$, yielding the average number of encounters per time step. This gives us a value that 
is meaningful and allows for comparison between runs with different memory lengths.

With the mechanics described, we can specify the model more formally, as follows:

1. Each of $N$ nodes is independently assigned a location, $\left(x_{i}, y_{i}\right)$, selected uniformly at random on a map of size $M$ (only one parameter is needed as we use a square map of area $M^{2}$ ).

2. Every pair of nodes, $(i, j)$, where $i \neq j$, is assigned a symmetric affinity, $a_{i j}=a_{j i}$, set equal to one with probability $A$, and zero otherwise.

3. For every pair of nodes, $(i, j)$, where $i \neq j$ and the distance separating them, $d_{i j}$, is not more than $R$, a connection is established by adding a single meeting at a randomly chosen point, $0<t<H$ in their interaction histories, $h_{i j}^{t}$ and $h_{j i}^{t}$, initialising the remainder of the history with 0 meetings.

4. For each simulated timestep $t<t_{\max }$ :

(a) For each pair of nodes, $(i, j)$, invite $j$ to $i$ 's gathering if $w_{i j}=w_{j i}>0$ meaning $j$ is a current network neighbour of $i$, and $a_{i j}=1$, and $d_{i j} \leq R$.

(b) For each node, $i$, accept at most $S$ invitations, each selected with probability proportionate to the edge weight $w_{i j}$.

(c) For each node, $i$, consider each pair of individuals $(j, k)$ attending $i$ 's gathering (including $i$ themselves), where $j \neq k$, and increment their interaction history by one meeting in the most recent time slot, i.e., increment $h_{j k}^{0}$ and $h_{k j}^{0}$ by one.

(d) For each node, $i$, update their interaction history by shuffling more recent values one step down the list and overwriting the oldest value 


$$
\begin{aligned}
& h^{H} \text {, i.e. } \\
& \forall t \in\{0,1, \ldots, H-1\}: h_{j k}^{t+1} \leftarrow h_{j k}^{t}
\end{aligned}
$$

\section{Topology and network dynamics}

In this section we will present results obtained from the model introduced above, analysing its dynamic behaviour and characterising the resulting networks and their properties.

The networks obtained are typically sparse, exhibiting both densely connected clusters and weaker bridges between clusters. Thus, the resulting networks exhibit community structure. An example network is shown in Figure 1 and in Table 1 we list the characteristic properties of networks generated with the standard parameter setting of $R=30, A=0.75, S=3, H=50$ and $M=200$. Each value shown is the rounded average taken over 100 runs, with the standard deviation shown in brackets. The values obtained for the statistical measures match those of real-world social networks with high clustering, positive assortativity with respect to degree and short characteristic path length (Newman, 2003).

\section{Figure 1 and Table 1 about here}

The degree distribution is relatively broad, spanning an order of magnitude, and centred around a characteristic value as has been observed for real-world social networks where links require maintenance (Amaral et al., 2000). The maximum degree has a cut-off, which has been suggested as another important characteristic of interaction networks (Figure 2) (Hamill and Gilbert, 2010).

\section{Figure 2 about here}

The network's properties scale appropriately. For instance, for a larger system consisting of $N=1000$ nodes (the size of the grid was increased appropriately to ensure the density of nodes remained the same for $N=100$ and 
$N=1000$ ), we observe the same structures as before, leading to comparable values of clustering, assortativity and average degree (see Table 1 for values and Figure 3 for an example network). Since these measures are linked strongly to the connectivity, they are mainly constrained by the spatial restrictions placed on the interactions. Therefore, the increased number of nodes does not result in growth of the existing structure but in the presence of more of the local structure previously observed. They are linked together to form a larger network, leading to increased diameter, shortest path length and number of components (see Figure 4). The increase in those measures is due to the fact that long range links are not possible in the model because of the restrictions on social reach, thus prohibiting the existence of edges bridging long distances.

\section{Figure 3 about here}

\section{Figure 4 about here}

The spatial constraints imposed by finite reach encourage the high clustering and community structure typical of a social network. However, the networks are not just random geometric graphs with threshold distance, $R$. Gatherings bring about longer range edges with a length up to $2 R$, but this means that these longer range edges can only be created if they close a triangle. These edges are able to persist only as long as connections to the shared neighbour that facilitated the edge creation continues to exist. Because of this dependence on other edges we will refer to these edges as secondary edges and to the edges with length less than $R$ (which can be directly maintained by mutual invitation) as primary edges. Since edges with length between $R$ and $2 R$ have to be maintained by a mutual friend, many nodes separated by such a distance are not connected (see Figure 5). The influence of affinity results in not all possible primary edges being present since as some individuals will not invite each other. Furthermore, the stochasticity of the meeting process in combination with the time constraint 
can lead to a primary edge being lost in which case it can only be recovered if the pair of nodes have at least one common neighbour.

\section{Figure 5 about here}

Comparing the weights of existing edges, the secondary edges that do exist tend to be weaker than the primary edges (see Figure 6). This stems from the fact that two individuals $i$ and $j$ at distance $R<d_{i j}<2 R$ are unable to reinforce their connection by attending each others' own gatherings and also that the increased distance between them ensures that they share fewer common neighbours.

\section{Figure 6 about here}

Regarding the dynamics of the system, the topological measures reach equilibrium after $H$ timesteps as shown in Figure 7. For this figure, the system is perturbed by removal of a large number of edges (200 edges) at step $t=1000$. The system recovers quickly after $H$ timesteps, with the measures returning to values close to their pre-perturbation values, demonstrating that the system dynamics are somewhat robust to external perturbation. Complete recovery from perturbation is not possible in the case where the removal of edges fractures the network into more components as the process of triangle closure cannot reconnect a component that has been split into two disconnected fragments. The observed increase in number of components after the perturbation shows that fragmentation of the network does indeed happen. The resulting decrease in degree is an obvious consequence as now fewer potential neighbours are available to some of the nodes. In contrast, the reduction in diameter and average shortest path length result from the way these measures are calculated - we measure the characteristic path length and the diameter considering only the shortest paths between pairs of nodes that lie within the same network component, rather than leaving these values undefined for networks that involve 
more than one component. Consequently, network fragmentation created by the perturbation will tend to permanently reduce these values.

\section{Figure 7 about here}

The system reaches a quasi-stable equilibrium in which the statistical properties of the topology do not change, whereas the exact topology described by edges and their weights is subject to random fluctuations. This is exemplified in Figure 6 where the change of the edges weights of all edges attached to a randomly selected node in a single run are shown. The edge weights fluctuate over time due to the stochasticity in the meeting process generated by limiting the number of invites accepted to $S$ and having only a finite memory of length $H$. The magnitude of the fluctuations can be controlled by changing $H$. A higher value (longer memory) means that the edge weights are calculated over a larger window of time, leading to less volatility (Figure 6).

\section{Exploring the Parameter Space}

The model has four main parameters that control network dynamics and, consequently, influence topology: the social reach, $R$; the probability of affinity, $A$; the maximum number of invitations accepted per time step, $S$; and the length of the interactions histories, $H$. In this section we explore the effect that varying these parameters has and determine the portion of parameter space for which the model produces networks with desired characteristics.

Low values of $H$ lead to more noise and greater fluctuation in the edge weight dynamics. This can be observed in Figure 6 which shows the dynamics of the edge weights of all edges attached to a particular node for one run where $H=50$ and another with $H=5$. The higher value of $H$ shows substantially smaller fluctuations. More fluctuation in the edge weights leads to more fragmentation events where a component is split in two. Since components cannot fuse, de- 
creasing $H$ leads to an increase in the number of components (see Figure 8). For very low values $(H=2)$ the network is fragmented into many components and displays almost maximal clustering since only fully connected cliques can persist in the presence of high noise.

\section{Figure 8 about here}

Conversely, increasing $H$ allows each node to keep track of an increased number of relationships, leading to and increase in the maximum and average degree and flattening the degree distribution (as shown in Figure 9). Sufficiently high values of $H$ allow nodes to remember enough neighbours for the system to transition from a collection of disconnected components where only small cliques with strong links can persist to a network with community structure where weak ties can exist as well.

\section{Figure 9 about here}

The parameter $R$ increases the social reach of each node, leading to more potential interactions. The length of network edges increases indicating that relationships are able to span greater distances. For low $R$, networks are sparse and fragmented into many components as each node has only a limited number of potential interaction partners (see top row of Figure 10). As $R$ increases, components connect until the network consists of one component. This point is reached at around $R=40$. At $R=30$ the majority of nodes belongs to a single connected component, however, some singleton nodes might still be present. At this point the diameter and characteristic path length are at their maximum, as the largest component spans the whole network (leading to a higher diameter than the average diameter of several smaller components) but the social reach is still too small to allow direct connections between pairs of nodes on opposite sides of the space. Note that this is the region where the previously increasing clustering decreases somewhat, as the previously isolated components connect 
without all triangles being able to close. Note that clustering stays fairly high in the system across all parameter settings shown, due to the triangle closure mechanism present (Figure 11).

\section{Figure 10 about here}

\section{Figure 11 about here}

Increasing the value of $R$ further weakens the influence of spatial proximity until networks eventually transition to random networks. As the model makes this transition, assortativity decreases until it becomes negative and clustering increases as more and more of the open triangles are no longer prevented from closing by spatial constraints on interactions. The value of $R$ at which the network transitions to a random network decreases for increasing $H$ (see Figure 12). This means that realistic looking social networks are obtained for intermediate values of the parameter $R$ of approximately $20<R<70$, with the exact range depending on the values of the other parameters as well as the desired density of the resulting social network.

\section{Figure 12 about here}

Increasing $R$ or $H$ leads to higher degree. Average node strength, however, is constrained by the parameter $S$. Figure 13 shows that increasing $R$ only increases the average strength up to a certain point at which it is limited by $S$.

\section{Figure 13 about here}

As mentioned, the maximum weight of each edge is $S+1$, therefore increasing $S$ increases the possible strength of the nodes. This effect is most obvious for the bottom row of Figure 10 where the edges are plotted thicker in proportion to their increasing weights. For $R=30$ which is the base parameter setting for the network presented here, increasing $S$ from 1 to 2 and then 3 first leads to an increase in degree. This increase in connectivity also leads to a decrease in the number of components. Increasing $S$ further to 4,5 and 6 has a much smaller 
effect on the degree as the number of nodes in reach is limited by $R=30$. Therefore, the increase in "socialising time" leads to an increase of the average weight as the extra time can not be spent on creating new relationships, so instead it is spent strengthening existing ones. (Figure 14)

\section{Figure 14 about here}

\section{Discussion}

We have presented a model that generates and maintains a weighted social network as a result of the interplay of local interactions, which have been chosen in a way to resemble human behaviour. The resulting network is dynamic and reaches a quasi-stable equilibrium in which global topology measures equilibrate. Locally, edges continue to change in weight, the magnitude of these fluctuations being controlled by the parameter $H$. We have discussed the importance of including a mechanism to balance edge formation and deletion and have shown that using a finite memory of past interactions provides an elegant way to both maintain a sparse network and generate meaningful, dynamic edge weights. This stands in contrast to existing models that use system-level events such as random node or edge removals that are not a direct result of individuals' decisions.

We have shown that some of the characteristic features of social networks can result from spatial constraints being placed on the interactions. In this model, the influence of the spatial constraint is controlled by the parameter $R$. Increasing $R$ produces a transition from almost empty networks (when most interactions are prohibited) to networks resembling social networks, eventually transitioning to random networks when spatial embedding ceases to be influ-

ential. Importantly, the range of $R$ for which networks exhibiting the defining features of social networks are obtained is reasonably broad and within that 
range, $R$ can be used to match the density of the network to a real-world setting. Furthermore, we have discussed how varying the amount of time available for maintaining connections $(S)$ influences the topology and regulates the weights and thus the strength of the nodes.

So far we have established that key constraints on social dynamics imposed by the model can bring about and then dynamically maintain key properties of social networks: restrictive spatial embedding $(R)$ achieves sparseness, clustering, assortativity, and community structure; the distribution of social affinity (A) allows for structural holes to be created as some triangles remain open; agent time budgets $(S)$ regulate the number and strength of relationships; and the time depth of agent social histories $(H)$ regulates the stability of local relationships. This bridging account, reflexively linking local processes to global system properties, establishes the model as a framework within which we can explore questions relating social network structure and dynamics.

For instance, we might concentrate on real-world social network properties that the model fails to generate: what must be added to the model in order to observe long-tailed degree distributions (which are typical only of social networks where relationships can be maintained at no cost), or complicated hierarchical community structure, or ongoing change in the identity of the highest degree nodes. The fact that the model has ongoing endogenous dynamics also makes it possible for the system to react to external perturbations. Therefore, the model could be used as a basis for studying the coevolutionary dynamics of social processes and social networks subject to exogenous influences such as birth, death, and changes in node properties such as affinity or location. Additionally, the model presents a dynamic network mechanism into which other sociological processes can be integrated, thus providing a framework to study coevolutionary interaction of a sociological process and network evolution. While it in its 
current form does not allow us to investigate questions about details of human social behaviour, it provides a basis for such exploration. In the future, we plan to extend this work to study how social networks can fragment as the result of an external perturbation, such as reversing a small number of affinity values.

To summarise, we have presented a model that captures the dynamics of a weighted social network using simple but plausible local behavioural rules governing the interactions between individuals. We have shown that the constraints placed on these interactions are crucial for the emergence of the topological features that characterise real-world social networks such as high clustering, community structure and assortativity. We have argued that the ongoing dynamics of the model make the model suitable as framework to study coevolutionary interaction of a sociological process and network evolution.

\section{References}

Amaral, L., Scala, A., Barthélémy, M., and Stanley, H. (2000). Classes of small-world networks. Proceedings of the National Academy of Sciences, 97(21):11149-11152.

Barnett, L., Di Paolo, E., and Bullock, S. (2007). Spatially embedded random networks. Physical Review E, 76(5):056115.

Barrat, A., Barthélemy, M., Pastor-Satorras, R., and Vespignani, A. (2004). The architecture of complex weighted networks. Proceedings of the National Academy of Sciences, 101(11):3747-3752.

Barrat, A., Barthélemy, M., and Vespignani, A. (2005). The effects of spatial constraints on the evolution of weighted complex networks. Journal of Statistical Mechanics, 2005:P05003. 
Boguñá, M., Pastor-Satorras, R., Díaz-Guilera, A., and Arenas, A. (2004). Models of social networks based on social distance attachment. Physical Review E, 70:056122.

Bravo, G., Squazzoni, F., and Boero, R. (2012). Trust and partner selection in social networks: An experimentally grounded model. Social Networks, 34(4):481-492.

Bryden, J., Funk, S., Geard, N., Bullock, S., and Jansen, V. (2011). Stability in flux: community structure in dynamic networks. Journal of The Royal Society Interface, 8(60):1031-1040.

Bullock, S., Barnett, L., and Di Paolo, E. (2010). Spatial embedding and the structure of complex networks. Complexity, 16(2):20-28.

Cattuto, C., Van den Broeck, W., Barrat, A., Colizza, V., Pinton, J., and Vespignani, A. (2010). Dynamics of person-to-person interactions from distributed rfid sensor networks. PloS One, 5(7):e11596.

Cummings, J., Lee, J., and Kraut, R. (2006). Computers, phones, and the Internet: Domesticating information technology, chapter Communication technology and friendship during the transition from high school to college, pages 265-278. Oxford University Press.

Davidsen, J., Ebel, H., and Bornholdt, S. (2002). Emergence of a small world from local interactions: Modeling acquaintance networks. Physical Review Letters, 88(12):128701.

Ebel, H., Davidsen, J., and Bornholdt, S. (2002). Dynamics of social networks. Complexity, 8(2):24-27.

Eguíluz, V., Zimmermann, M., Cela-Conde, C., and San Miguel, M. (2005). 
Cooperation and the emergence of role differentiation in the dynamics of social networks1. American Journal of Sociology, 110(4):977-1008.

Grabowski, A. (2007). Interpersonal interactions and human dynamics in a large social network. Physica A, 385(1):363-369.

Granovetter, M. (1973). The strength of weak ties. American Journal of Sociology, 78:1360-1380.

Gross, T. and Blasius, B. (2008). Adaptive coevolutionary networks: a review. Journal of The Royal Society Interface, 5(20):259-271.

Hamill, L. and Gilbert, N. (2009). Social circles: A simple structure for agentbased social network models. Journal of Artificial Societies and Social Simulation, 12(2):3.

Hamill, L. and Gilbert, N. (2010). Simulating large social networks in agentbased models: A social circle model. Emergence: Complexity and Organization, 12(4):78-94.

Herrmann, C., Barthelemy, M., and Provero, P. (2003). Connectivity distribution of spatial networks. Physical Review E, 68(2):026128.

Holme, P. and Kim, B. (2002). Growing scale-free networks with tunable clustering. Physical Review E, 65(2):026107.

Holme, P. and Newman, M. (2006). Nonequilibrium phase transition in the coevolution of networks and opinions. Physical Review E, 74(5):056108.

Illenberger, J., Nagel, K., and Flötteröd, G. (2012). The role of spatial interaction in social networks. Networks and Spatial Economics, pages 1-28.

Kumpula, J., Onnela, J., Saramäki, J., Kaski, K., and Kertész, J. (2007). 
Emergence of communities in weighted networks. Physical Review Letters, 99:228701.

Leskovec, J. and Horvitz, E. (2008). Planetary-scale views on a large instantmessaging network. In Proceeding of the 17th International Conference on World Wide Web, pages 915-924. ACM.

Levins, R. (1966). The strategy of model building in population biology. American Scientist, 54:421-431.

Liljeros, F., Edling, C., Amaral, L., Stanley, H., and Åberg, Y. (2001). The web of human sexual contacts. Nature, 411(6840):907-908.

Marsili, M., Vega-Redondo, F., and Slanina, F. (2004). The rise and fall of a networked society: A formal model. Proceedings of the National Academy of Sciences, 101(6):1439-1442.

Miritello, G., Moro, E., Lara, R., Martínez-López, R., Belchamber, J., Roberts, S., and Dunbar, R. (2013). Time as a limited resource: Communication strategy in mobile phone networks. Social Networks, 35:89-95.

Newman, M. (2002). Assortative mixing in networks. Physical Review Letters, $89(20): 208701$.

Newman, M. (2003). The structure and function of complex networks. SIAM review, 45(2):167-256.

Newman, M. and Park, J. (2003). Why social networks are different from other types of networks. Physical Review E, 68(3):036122.

Newman, M. E. J. (2010). Networks: An Introduction. Oxford University Press.

Onnela, J., Saramäki, J., Hyvönen, J., Szabó, G., Lazer, D., Kaski, K., Kertész, J., and Barabási, A. (2007). Structure and tie strengths in mobile com- 
munication networks. Proceedings of the National Academy of Sciences, 104(18):7332-7336.

Oreskes, N., Shrader-Frechette, K., and Belitz, K. (1994). Verification, validation, and confirmation of numerical models in the earth sciences. Science, 263(5147):641-646.

Penrose, M. (2003). Random Geometric Graphs, volume 5. Oxford University Press.

Skyrms, B. and Pemantle, R. (2000). A dynamic model of social network formation. Proceedings of the National Academy of Sciences, 97(16):9340-9346.

Sutcliffe, A., Dunbar, R., Binder, J., and Arrow, H. (2012). Relationships and the social brain: Integrating psychological and evolutionary perspectives. British journal of psychology, 103(2):149-168.

Szell, M. and Thurner, S. (2010). Measuring social dynamics in a massive multiplayer online game. Social Networks, 32(4):313-329.

Takács, K., Janky, B., and Flache, A. (2008). Collective action and network change. Social Networks, 30(3):177-189.

Toivonen, R., Onnela, J., Saramäki, J., Hyvönen, J., and Kaski, K. (2006). A model for social networks. Physica A, 371:851-860.

Van Segbroeck, S., Santos, F. C., Lenaerts, T., and Pacheco, J. M. (2009). Reacting differently to adverse ties promotes cooperation in social networks. Physical Review Letters, 102(5):058105.

Vázquez, A. (2003). Growing network with local rules: Preferential attachment, clustering hierarchy, and degree correlations. Physical Review E, 67:056104. 
Wong, L., Pattison, P., and Robins, G. (2006). A spatial model for social networks. Physica A, 360(1):99-120.

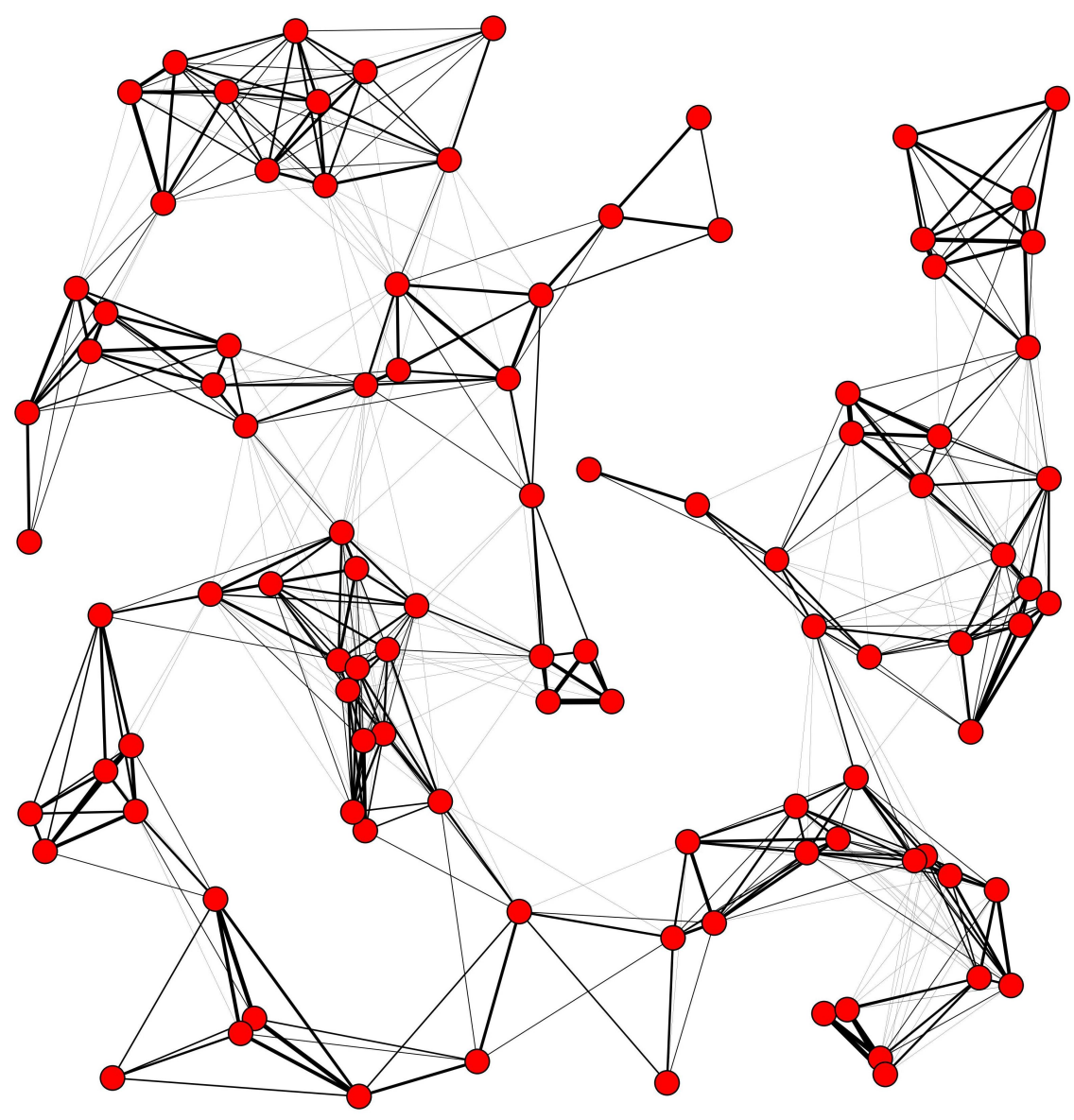

Figure 1: An example network at $t=10,000$ for the standard parameter setting $R=30, S=3, H=50, A=0.75$, and $N=100$ on a map of size $M=200$. The network exhibits community structure with densely connected components, which are linked together by weaker edges. 


\begin{tabular}{|l|rl|rl|}
\hline & $\mathrm{N}=100$ & & $\mathrm{~N}=1000$ & \\
\hline nodes* & 98.49 & $( \pm 1.23)$ & 992.06 & $( \pm 3.41)$ \\
edges & 535.56 & $( \pm 56.76)$ & 6153.70 & $( \pm 163.57)$ \\
clustering & 0.75 & $( \pm 0.04)$ & 0.71 & $( \pm 0.01)$ \\
$\quad$ weighted & 0.25 & $( \pm 0.03)$ & 0.20 & $( \pm 0.01)$ \\
transitivity & 0.69 & $( \pm 0.04)$ & 0.66 & $( \pm 0.01)$ \\
assortativity & 0.49 & $( \pm 0.11)$ & 0.55 & $( \pm 0.05)$ \\
shortest path & 3.49 & $( \pm 0.75)$ & 12.16 & $( \pm 1.78)$ \\
diameter & 8.49 & $( \pm 2.23)$ & 31.69 & $( \pm 5.89)$ \\
degree & 10.88 & $( \pm 1.17)$ & 12.41 & $( \pm 0.34)$ \\
strength & 12.22 & $( \pm 0.37)$ & 12.67 & $( \pm 0.09)$ \\
components & 2.88 & $( \pm 1.39)$ & 8.37 & $( \pm 2.96)$ \\
\hline
\end{tabular}

Table 1: Measures characterising the topology of the resulting networks for network sizes $N=100$ and $N=1000$, showing mean (over 100 runs) and standard deviation for each. $\left({ }^{*}\right.$ When calculating these measures singletons are ignored, meaning only nodes with at least one edge are considered. Nodes denotes the number of nodes that were not singletons and therefore included in the calculation.)

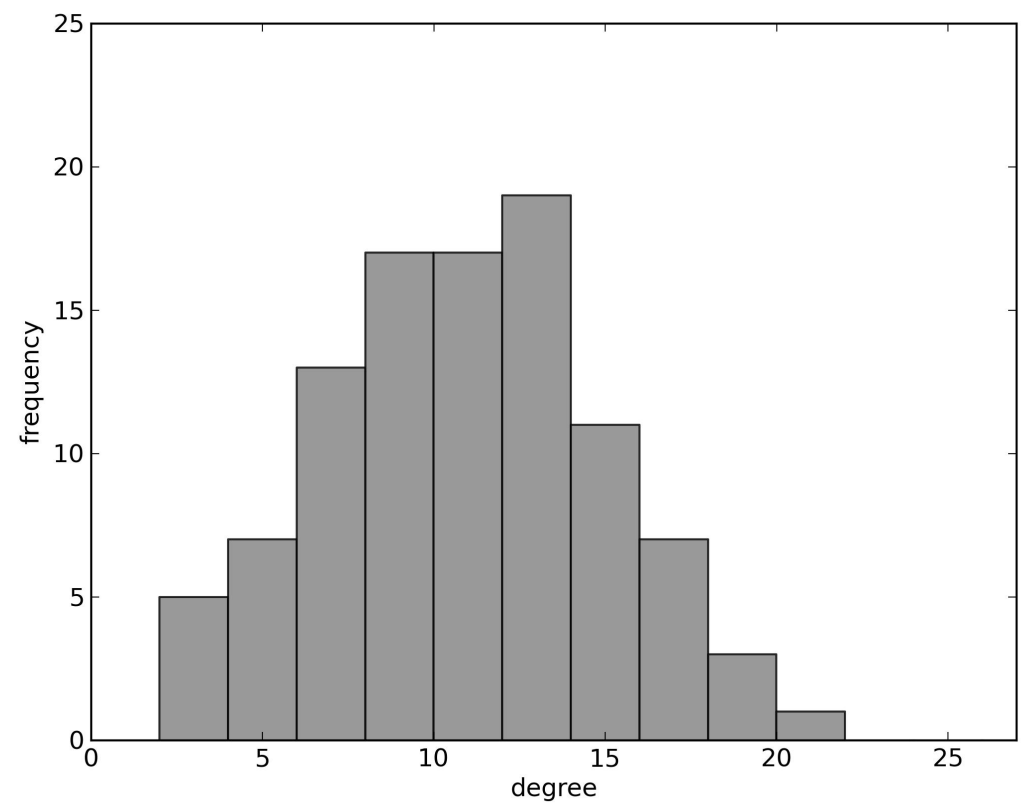

Figure 2: The degree distribution of the network shown in Figure 1. The distribution is broad, spanning an order of magnitude and limited for higher values, as observed in real social networks. 


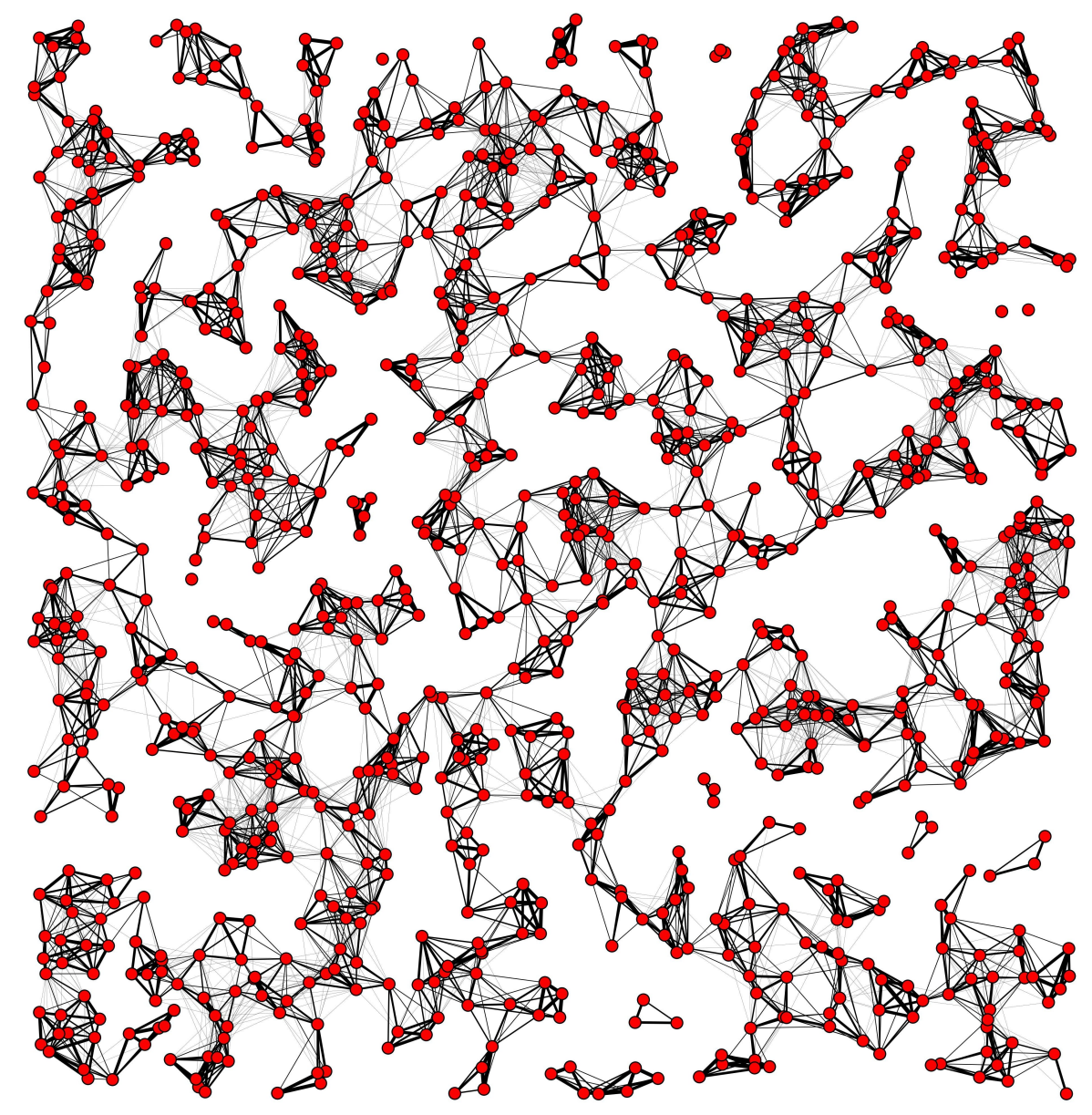

Figure 3: An example network with many nodes. Parameters are the same as in Figure 1 except for the increased number of nodes $N=1000$ and the size of the map being enlarged to $M=632$ to ensure the same node density as for the run with fewer nodes. The resulting network exhibits the same characteristic local structures as for $N=100$ but inevitably has a larger diameter and longer shortest path length due to longer range edges being prohibited by the spatial constraint (see Table 1). 


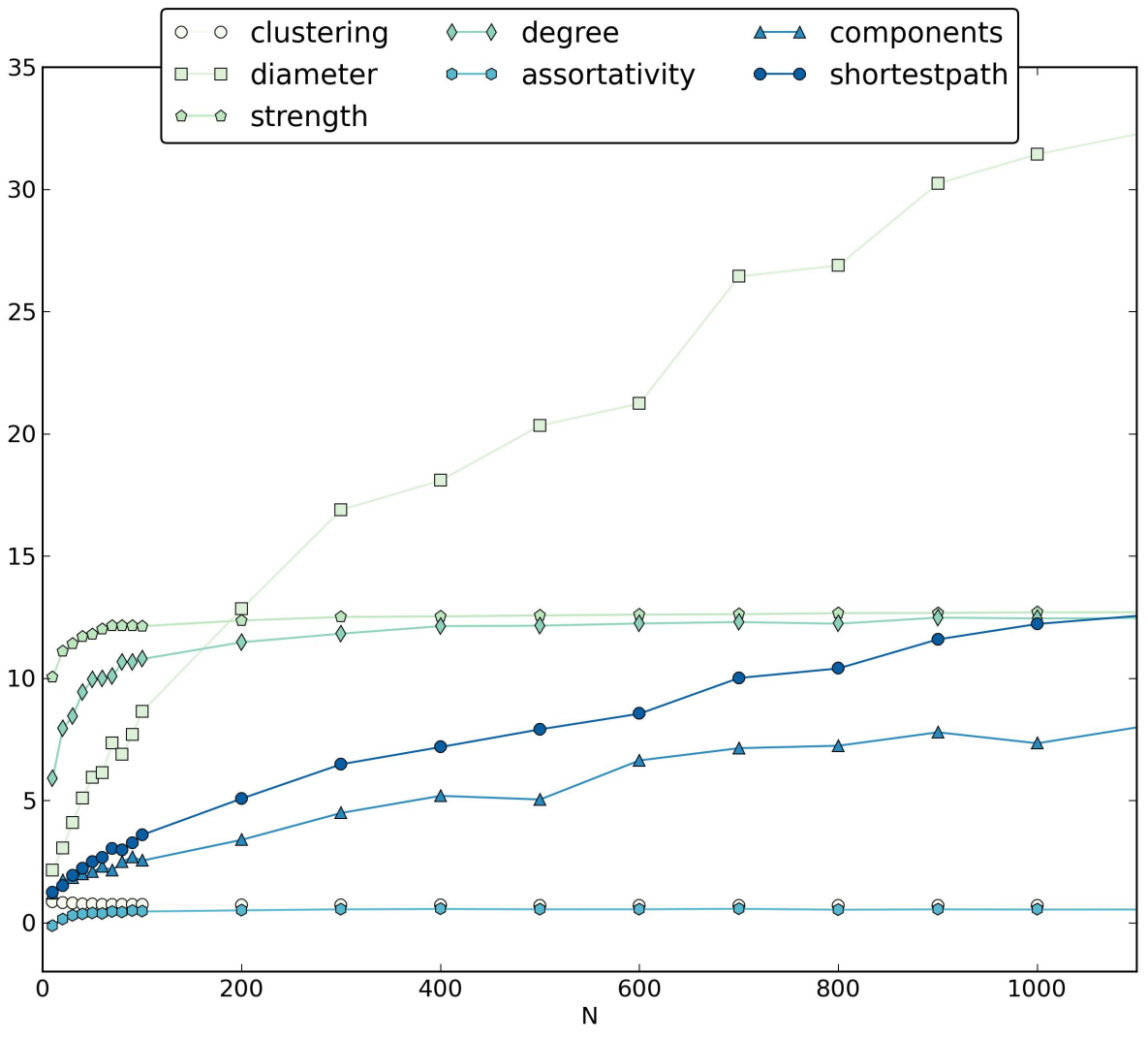

Figure 4: Topological measures for different network sizes $N$. Clustering, strength and average degree and assortativity do not scale with $N$ (apart from for small values of $N$ ) while diameter, average shortest path length and the number of components increase with increasing $N$. All values shown are averages over 20 runs. The map was enlarged with increasing $N$ to keep the node density constant across different values of $N$ according to $M=\sqrt{400 \cdot N}$, all other parameters were kept constant to the standard parameter setting of $R=30$, $A=0.75, S=3$, and $H=50$. 


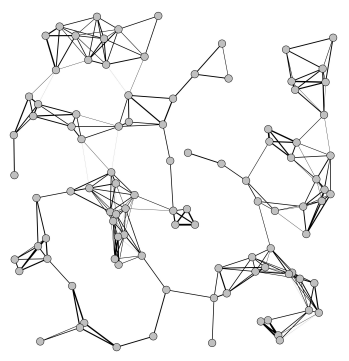

(a) Primary edges present

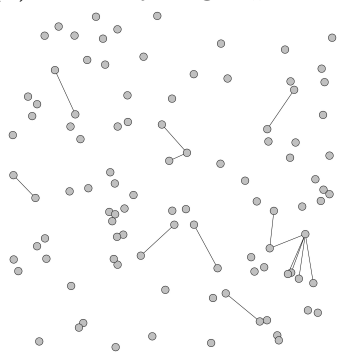

(c) Primary edges missing

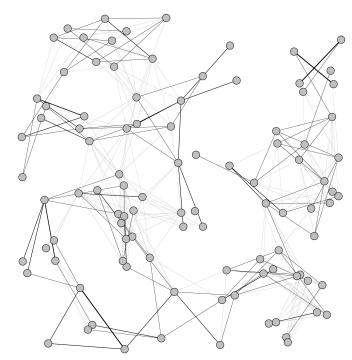

(b) Secondary edges present

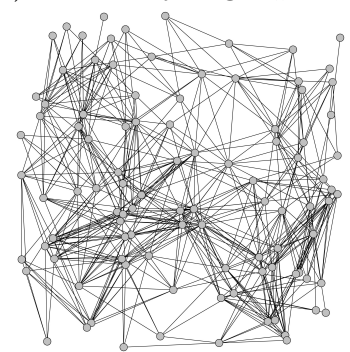

(d) Secondary edges missing

Figure 5: Classes of edges within the network shown in Figure 1. Primary edges are between nodes close enough to invite each other to gatherings (i.e. $d_{i j} \leq R$ ). Secondary edges are between nodes that may only interact at the gatherings of mutual neighbours (i.e. $R<d_{i j} \leq 2 R$ ). A typical network features many but not all of the possible primary edges and a much smaller fraction of the possible secondary edges. 


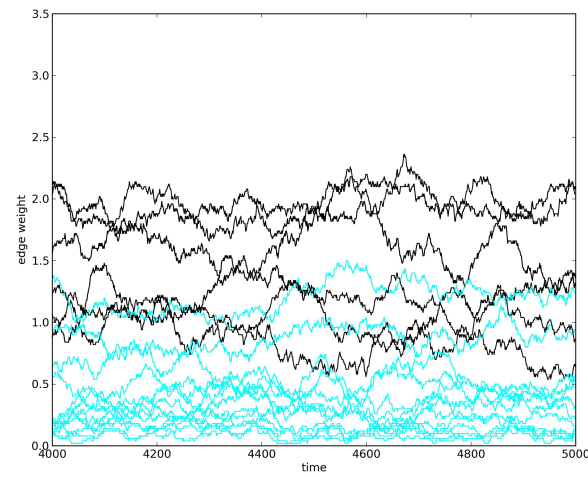

(a) $H=50$

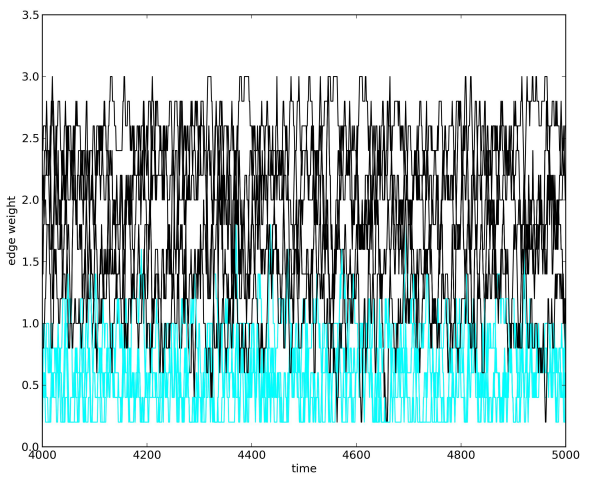

(b) $H=5$

Figure 6: Edge weights over time for all edges attached to a randomly chosen node, shown for the interval $t=4000$ to $t=5000$ where the system has reached global equilibrium where topological measures are stable but individual weights continue to fluctuate. Primary edges are plotted in black, secondary edges in a lighter shade (cyan). To highlight the effect of the history parameter $H$ on the stochastic fluctuations the settings $H=5$ and $H=50$ are shown, displaying a much larger magnitude and frequency in fluctuations for shorter history dynamics. All other parameters were set as in Figure 1 


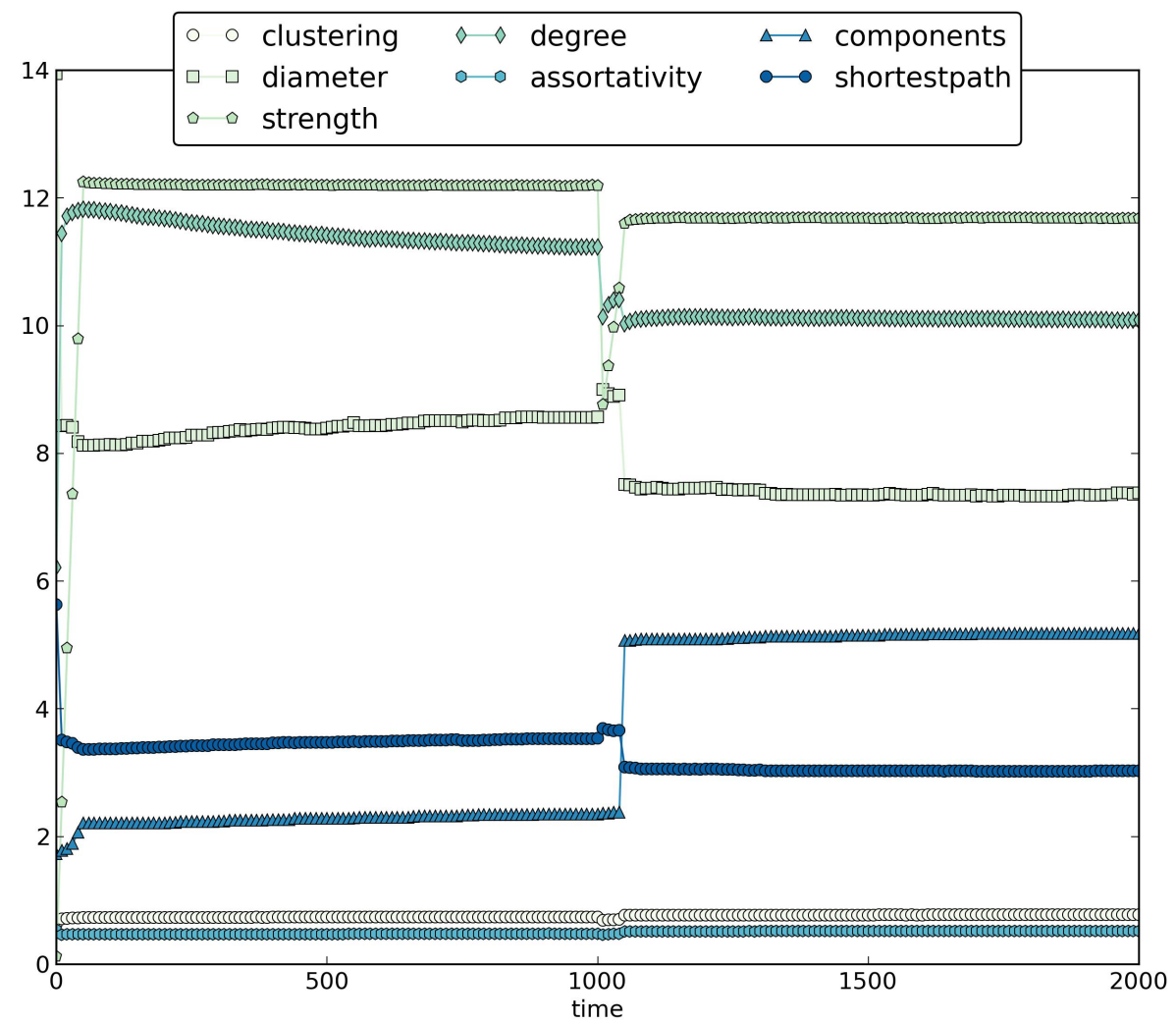

Figure 7: Topological measures equilibrate rapidly, within $H$ timesteps after which initialisation effects have been removed. The measures recover following disturbance at $t=1000$ where 200 randomly selected edges are removed. The parameters used were $R=30, S=3, H=50, A=0.75$, and $N=100$, measures are means taken over 100 runs. 


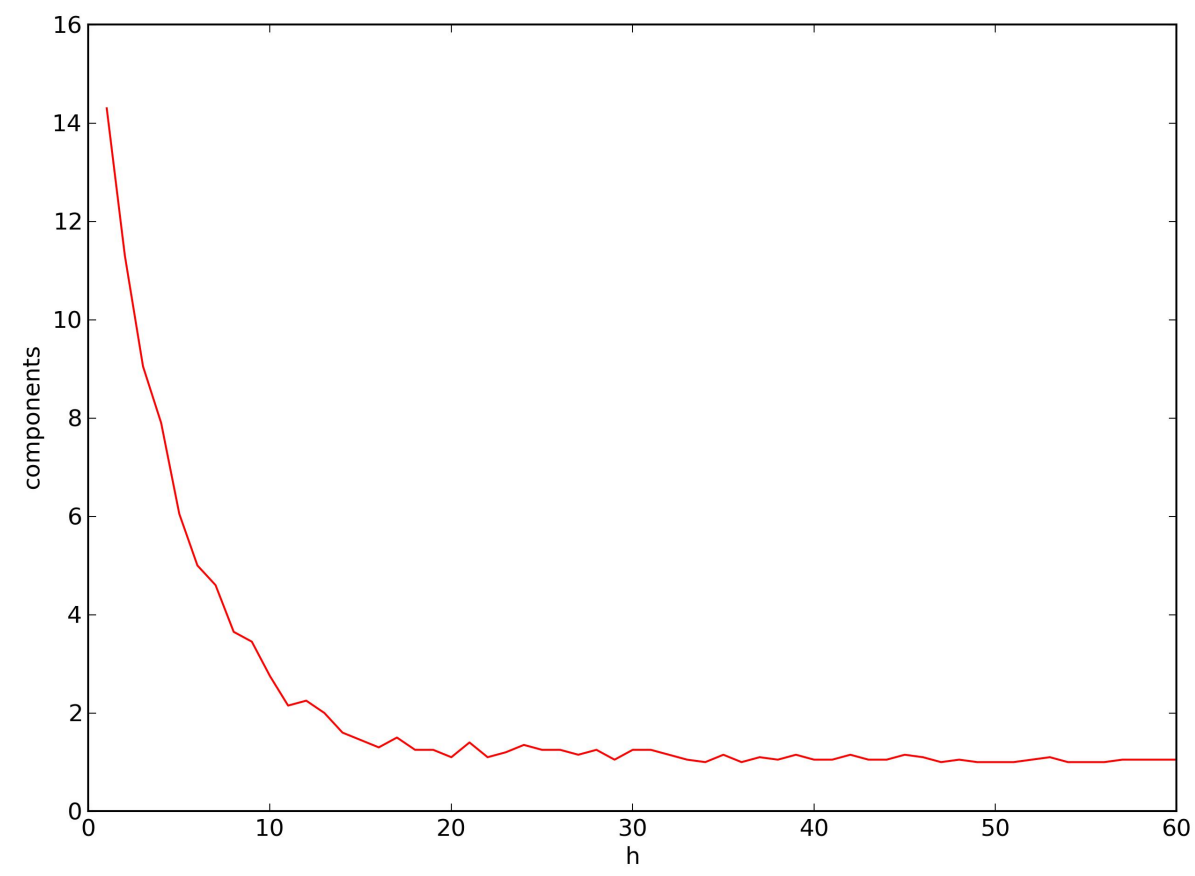

Figure 8: Number of components decreasing for increasing values of $H$ (memory length). Parameters used are $R=50, A=0.75, S=3$ and $M=200$, with 20 repeats per data point. Note that in contrast to Table 1, the calculation of the number of components does include singletons.

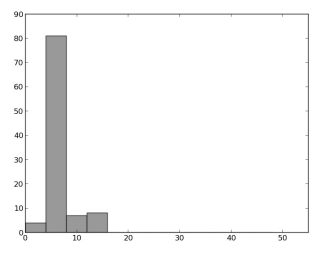

(a) $H=2$

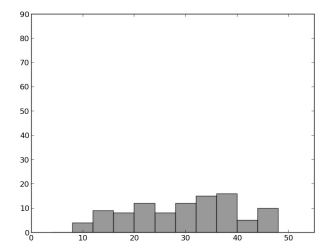

(d) $H=16$

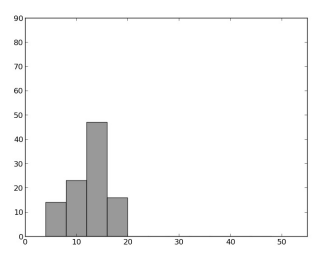

(b) $H=4$

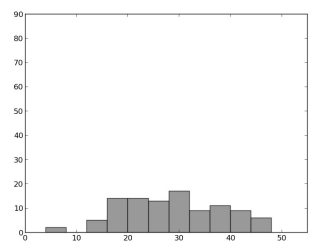

(e) $H=32$

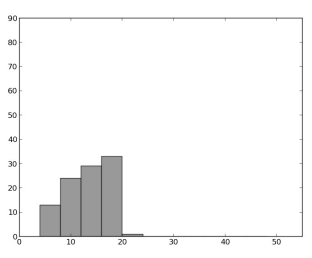

(c) $H=8$

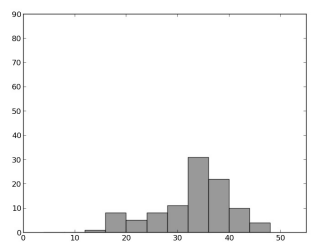

(f) $H=50$

Figure 9: Degree distributions for different values of $H=2,4,8,16,32,50$. Parameters are per Figure 8. 


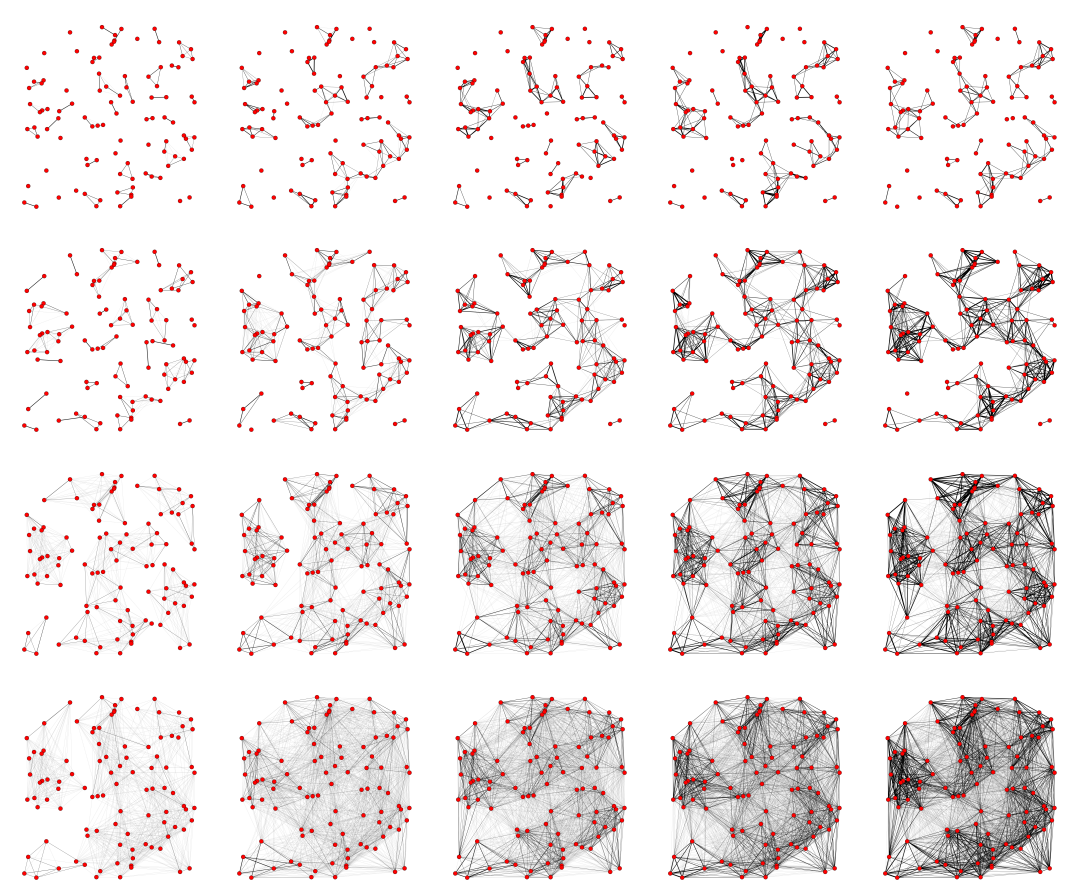

Figure 10: Resulting networks after $t=10000$ steps for $H=50, A=0.75$ and varying $R$ (rows, from top to bottom: $R=20,30,50,70$ ) and $S$ (columns, from left to right: $S=1,2,3,4,5)$, using the same placement of nodes for all runs. 


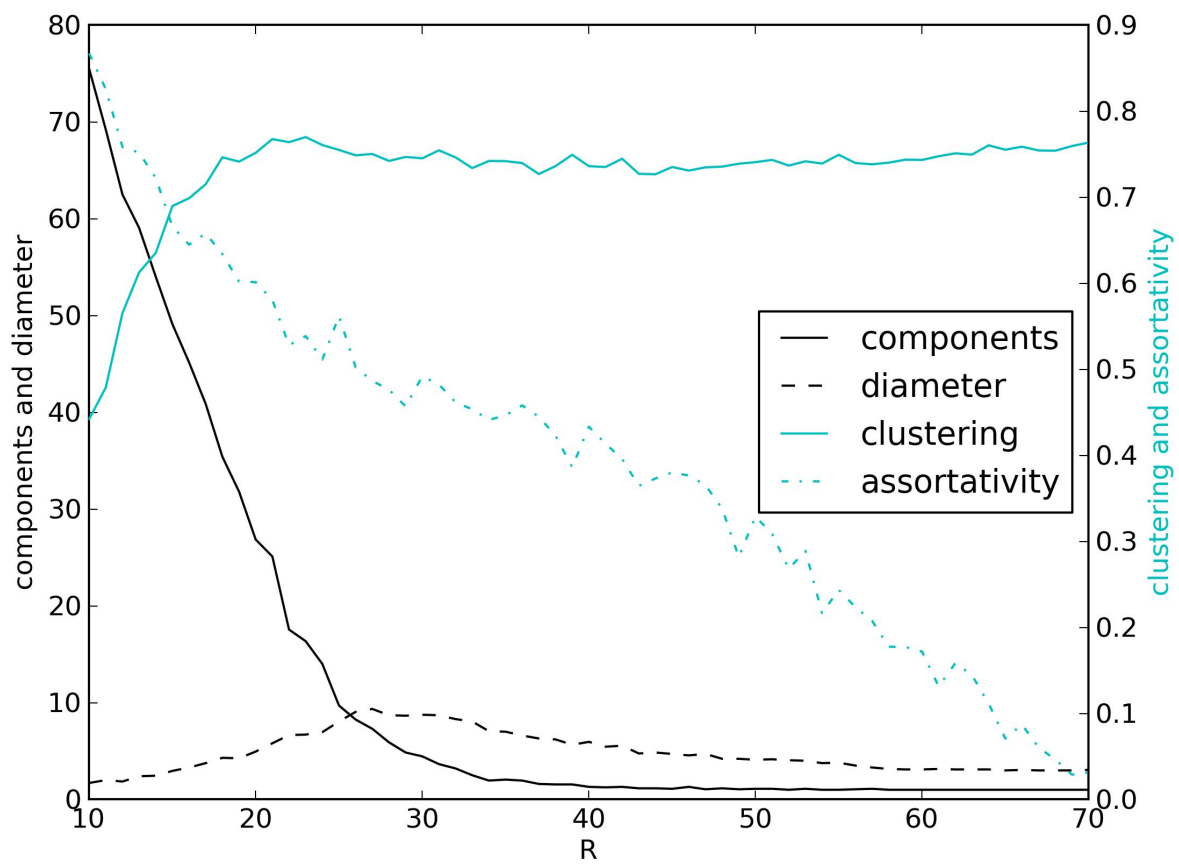

Figure 11: Topological measures changing in reaction to varying the social reach $R$ ( $R$ ranging from 10 to 70,20 repeats per data point). Note that in contrast to Table 1, the calculation of the number of components does include singletons as we are considering parameter settings for which significant numbers of singletons exist. Other parameters where set as in Figure 1. 


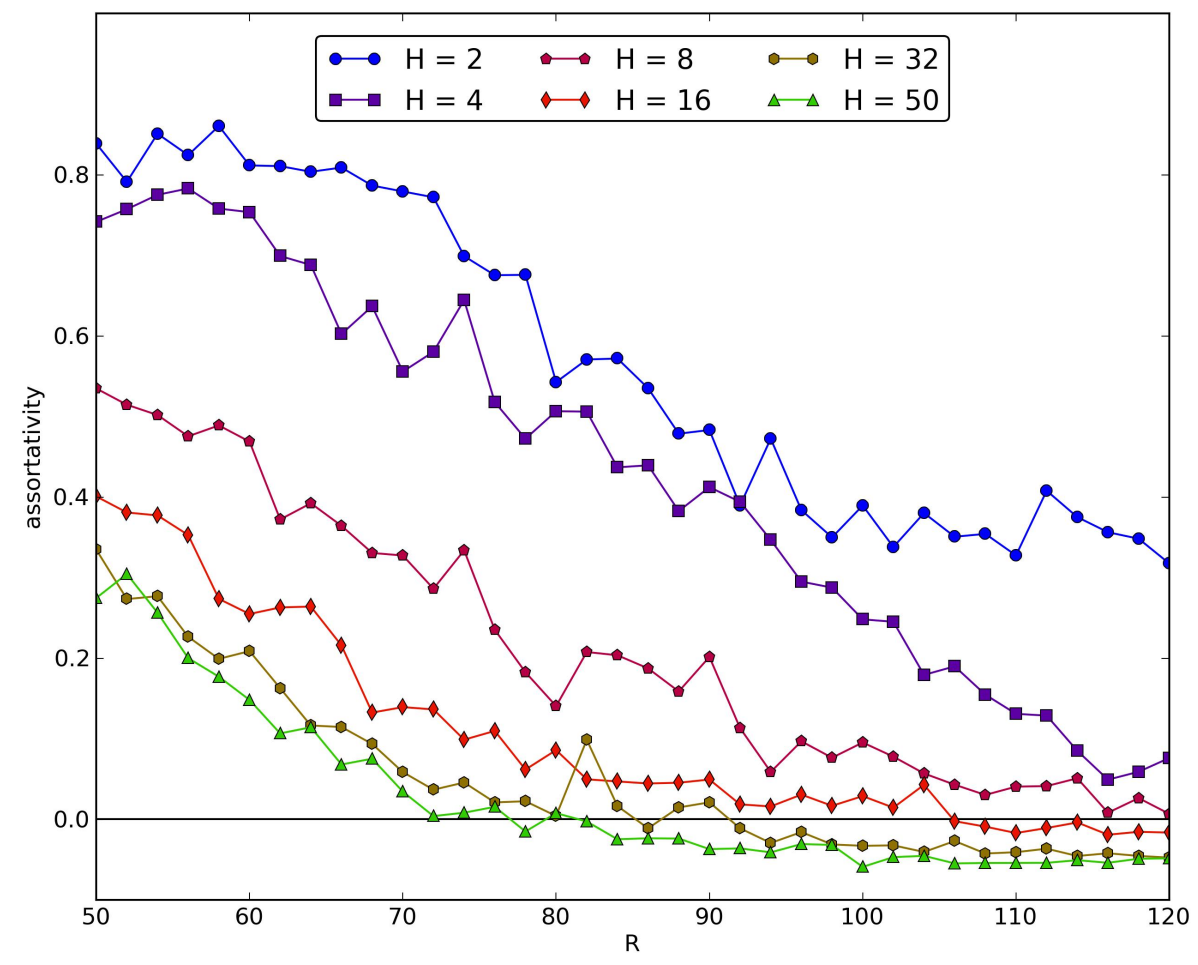

Figure 12: Mean of assortativity (calculated over 20 runs per data point) showing that the value of $H$ influences the point at which the network transitions towards a random network, crossing to negative values of assortativity. $R$ ranging from 50 to 120 , shown for different values of $H$. $S$ and $A$ were set to the standard parameter setting of $S=3$ and $A=0.75$. 


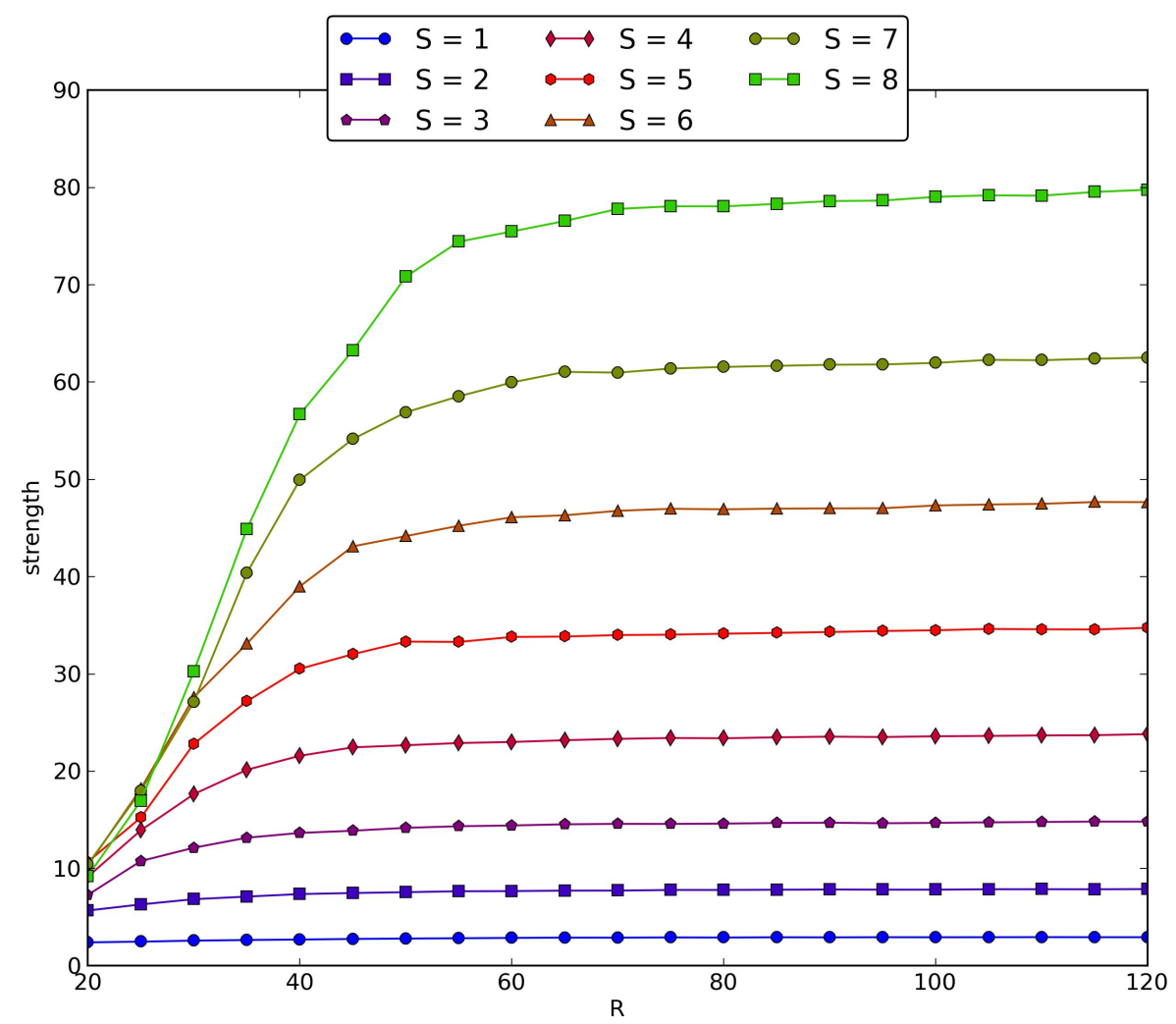

Figure 13: Strength in reaction to varying $R$ for different values of $S, 20$ repeats for each data point, $H=50, A=0.75$. The average strength increases for increasing $R$, with the maximum value reached being determined by the number of slots available per timestep $(S)$. 


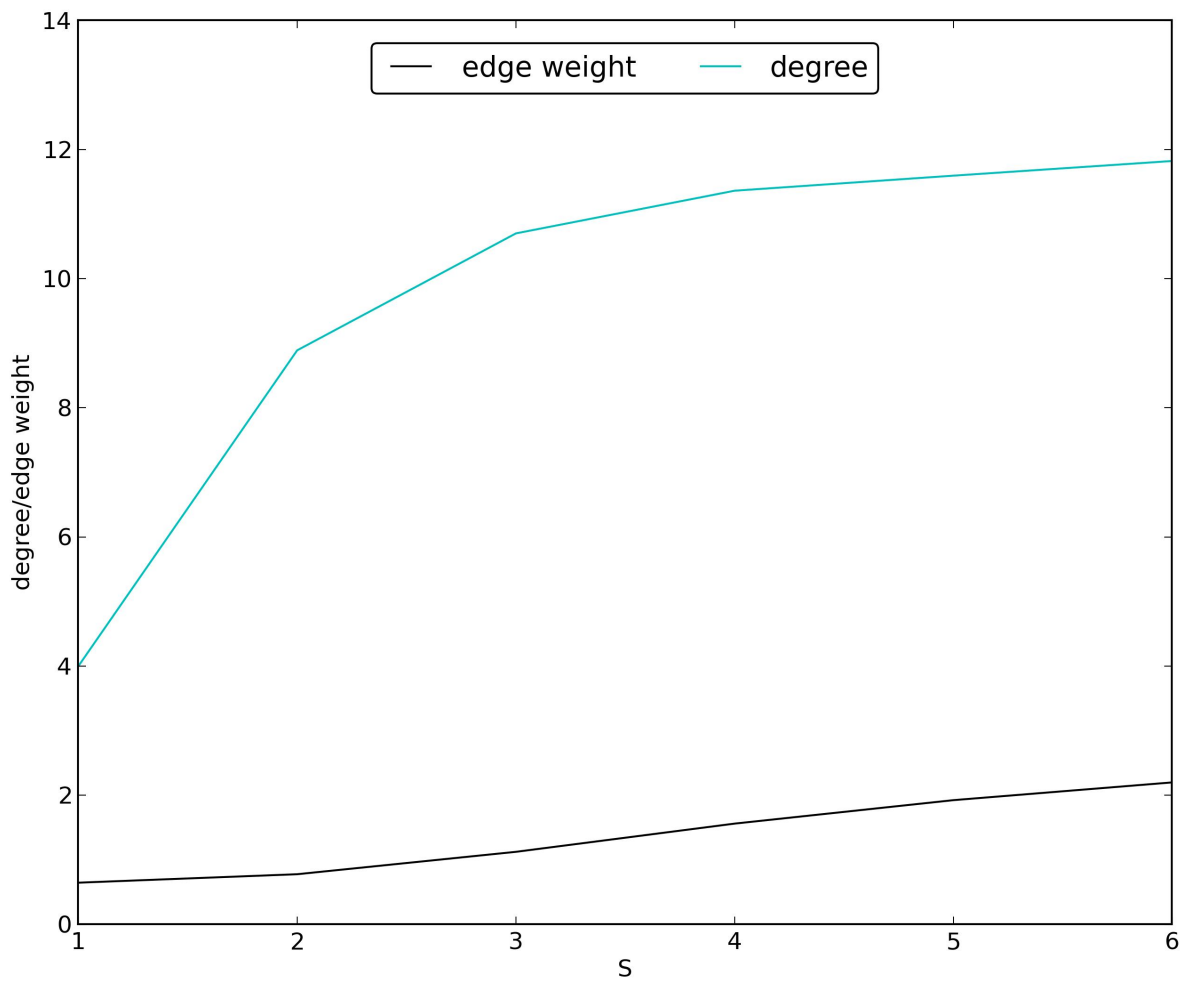

Figure 14: Mean of average degree and edge weight (calculated over 20 runs per data point) showing that increasing $S$ initially leads to an increase in degree and later (for $S>3$ ) to increased average edge weight. Parameters used were $R=30, A=0.75$ and $H=50$. 